\title{
The Effect of Cesarean Delivery on Child Survival and Early Breastfeeding Practices: Global Data from 103 Provinces in the Eastern Mediterranean Region
}

\author{
Ayoub Al-Jawaldeh, Azza Abul-Fadl \\ Associate; Department of Nutritional Sciences; Faculty of Life Sciences, University of Vienna; \\ Address: Althan-Str. 14; 1090 Vienna, Austria \\ Professor of Pediatrics, Faculty of Medicine, Pediatric department, Benha University \\ Address: 26 B ElGezira Al Wosta street, Zamalek Cairo, Egypt
}

\begin{abstract}
Background: Early initiation of breastfeeding (EIBF) plays an important role in the reduction of infant mortality. Although delivery by cesarean section (CS) can save lives but delays EIBF and thereby increases risk of child mortality.
\end{abstract}

Aim: To examine the relationship between CS and EIBF with child mortality.

Methods: Data was recruited from recent demographic health surveys for 129 provinces of seven Eastern Mediterranean region (EMR) countries. Data for EBFI was available in 103 provinces. All data was analyzed for CS in relation to EIBF in first hour and first day, feeding prelacteals, delivery by skilled birth attendant (SBA), neonatal mortality (NMR), infant mortality (IMR) and under-five mortality rates (U5MR). The economic cost of unnecessary CS was analyzed. Statistical analysis was done using Pearson correlation and descriptive statistics.

Results: CS was inversely associated with EIBF in the first hour after birth $(\mathrm{r}-0.2, \mathrm{P}=0.1)$, intake of prelacteals $(\mathrm{r}-0.3, \mathrm{P}=0.008)$ and NMR ( $\mathrm{r}-0.3, \mathrm{P}=0.1)$, IMR ( $\mathrm{r}-0.4, \mathrm{P}=0.0001)$, and U5MR ( $\mathrm{r}-0.4$, $\mathrm{P}=0.0001)$ at $\mathrm{P}<0.05$, and positively associated with $\mathrm{EIBF}$ in first day $(\mathrm{r} 0.2, \mathrm{P}=0.06)$. CS below the recommended level by WHO $(<15 \%)$ was inversely associated with IMR. Moderately high CS $>18 \%-$ $38 \%$ was inversely correlated with EIBF and positively correlated with intake of prelacteals, NMR and IMR. Unnecessary CS and cumulative cost of CS was significantly correlated with U5MR, IMR and NMR $(\mathrm{P}<0.000)$.

Conclusions: Moderately high and especially unnecessary CS by unskilled birth attendant increase child mortality in the EMR. Efforts to increase breastfeeding initiation, as early as possible through skin-toskin, with no prelacteals, may help alleviate the mortality rates from CS.

Key words: cesarean-section delivery, initiation of breastfeeding, neonatal mortality, infant mortality, prelacteal feeds.

\section{Introduction}

Researchers have shown that women who deliver by cesarean section (CS) are less likely to breastfeed, or delay breastfeeding initiation ${ }^{(1)}$. Breastfeeding within the first hour post-delivery has been cited as an important predictor of continued breastfeeding ${ }^{[2]}$. A longitudinal follow-up study of women in China who had a CS delivery showed a lower rate of exclusive breastfeeding (EBF) and any breastfeeding than those who had a vaginal delivery ${ }^{(3)}$. In addition, CS delivery was related with formula feeding in the hospital and delayed breastfeeding initiation ${ }^{(3)}$ and this interferes with breastfeeding continuity ${ }^{(4)}$. However a study conducted in Egypt ${ }^{(5)}$ showed that when women delivered by CS are supported in early breastfeeding initiation (EIBF) through skin-to-skin contact (SSC) for at least 20-30 minutes and follow-up support in breastfeeding, the continuity rates of breastfeeding were similar to those who underwent vaginal delivery. 
Another study showed that women who were provided with support in early SSC were more likely to initiate and continue breastfeeding ${ }^{(6)}$. Furthermore EIBF with SSC has been shown to decrease neonatal and infant mortality ${ }^{(7)}$.

However there is an argument of whether the 15\% upper limit for CS, suggested by the World Health organization (WHO) in 1985, could be less valid nowadays taken into account changes of the population in high income countries, such as mother's age at the first child, birth-weight and other factors that may result in needing more or less CS. However, studies have shown that until now there is no evidence of benefit for the health of mothers and babies in populations with values of CS above $15 \%{ }^{(8)}$. Regarding the lower limit, it has been argued that CS rates of $5 \%$ could achieve major improvement on maternal outcomes ${ }^{(9)}$. However, for neonatal health and survival, CS rates below 5\% may be harmful, whereas CS rates between $5 \%$ and $10 \%$ have been reported to attain better outcomes ${ }^{(10,11,12)}$.

We propose, as our null hypothesis, that the controversy of whether delivery by CS can influence child survival can be linked to EIBF. Since delay in breastfeeding initiation can increase mortality and CS can also influence mortality, it is not clear why CS can reduce child mortality in some instances but increases it in others. Suboptimal breastfeeding initiation, prelacteals, supplements given at birth, interference with first hour SSC may play a role in clarifying these controversies. Hence the aim of this study is to analyze country data from demographic surveys to achieve a better understanding of the underlying determinants that can improve poor outcomes of CS delivery and in the same time improve breastfeeding practices that can influence child survival in the Eastern Mediterranean region (EMR).

\section{Methods}

Data source: The data was assembled from the demographic health surveys (DHS) and Multiple Indicator Cluster Survey (MICS) and included data related to EIBF in the first hour and EIBF within the first day i.e. $<0-23$ hours of life (EIBF< 24hrs), feeding prelacteals and skin to skin whenever recorded. Data about assistance at delivery included skilled birth attendant (SBA) at delivery, cesarean section delivery (CS) rates, and attendance of antenatal care (ANC) for over 4 times or any ANC in countries where the latter data was not available as in Yemen.

Countries and provinces: We studied data from demographic health surveys (DHS) of seven countries: Afghanistan in $2015^{(13)}$, Egypt in $2014^{(14)}$, Jordan 2017-18 ${ }^{(15)}$, Pakistan 2017-18 ${ }^{(16)}$, Sudan ${ }^{(17)}$, Multiple Indicator Cluster Survey (MICS) of Somalia North East (NE) in $2011^{(18)}$ and MICS of Somaliland in $2011^{(19)}$ and finally Yemen in $2013^{(20)}$. The provinces (regions or governorates) included 33 in Afghanistan, 25 in Egypt, 12 in Jordan, 13 in Pakistan, 3 regions in Somalia NE and 5 regions in Somaliland, 18 in Sudan, 21 in Yemen, totaling 130. The provinces (regions or governorates) for each country included:

In Afghanistan (33): Kabul, Kapisa, Parwan, Wardak, Logar, Nangarhar, Laghman, Panjsher, Baghlan, Bamyan Ghazni , Paktika, Paktya, Khost, Kunarha, Nooristan, Badakhshan, Takhar, Kunduz, Samangan, Balkh, Sar-E-Pul, Ghor, Daykundi, Urozgan, Kandahar, Jawzjan, Faryab, Helmand, Badghis, Herat, Farah, and Nimroz.

Egypt governorates (25): Cairo, Alexandria, Port Said, Suez, Damietta, Dakahlia, Sharkia, Kalyubia, Kafr El-Sheikh, Gharbia, Menoufia, Behera, Ismailia, Giza, Beni Suef, Fayoum, Menya, Assuit, Souhag, Qena, Aswan, Luxor, Red Sea, New Valley and Matroh (North and South Sinai not included).

Jordan governorates (12): Amman, Balqa, Zarqa, Madaba, Irbid, Mafraq, Jarash, Ajloun, Karak, Tafiela, Ma'an, Aqaba.

Pakistan regions (12): Punjab (urban and rural), Sindh (urban and rural), Khyber Pakhtunkhwa (urban and rural), Balochistan (urban and rural) and ICT Islamabad and FATA, Azad Jammu and Kashmir (urban and rural) and Gilgit Baltistan,

Somalia regions (8): Somaliland included 5 regions: Maroodijeex/Saaxil, Awdal, Togdheer, Sool and Sanaag and Somalia NE included 3 regions: Bari, Nugal and Mudug.

Sudan governorates (18): Northern, River Nile, Red Sea, Kassala, Gadarif, Khartoum, Gezira, White Nile, Sinnar, Blue Nile, North Kordofan South Kordofan, West Kordofan, North Darfur, West Darfur, South Darfur, Central Darfur, East Darfur. 
Yemen governorates (21): Ibb, Abyan, Sana'a City, Al-Baidha, Taiz, Al-Jawf, Hajjah, Al-Hodiedah, Hadramout, Dhamar, Shabwah, Sadah Sana'a, Aden, Lahj, Mareb, Al-Mhweit, Al-Mhrah, Amran, Aldhalae, Reimah.

The recent UNICEF MICS programme used similar modules to the DHS making comparisons possible. The surveys from which data was compiled were conducted by a national body and assisted by USAID and other donor agencies which provide assistance to the government. The data retrieved from the most recent national demographic surveys were compiled and analysed using statistical methods for analysis including the correlative studies using Pearson's correlative analysis. The cut off for statistical significance used was $\mathrm{P}<0.05$.

\section{Results}

Table (1) presents the mean and standard deviation for the percent for EIBF in first hour and first day, prelacteal feeds, delivery by skilled health professionals, delivery by CS, NMR per thousand live births and IMR per thousand live births in the provinces of the seven countries. Mean \% EIBF in the 1st hour and first day for all 101 provinces of six countries (Egypt excluded) was 55.9 \pm 18.9 and $69.01 \pm 20.9$ respectively.

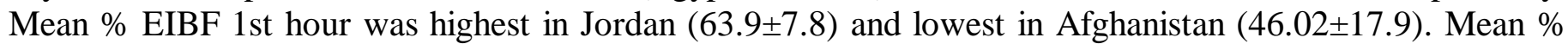
EIBF 1st day was highest in Afghanistan (91.7 \pm 7.2$)$ and lowest in $(46.02 \pm 17.9)$. Mean \% prelacteal feeds offered in 101 provinces in the region was $63.3 \pm 26.5$ being highest in Yemen $65.5 \pm 12.5$ and lowest in Sudan 27.4 \pm 11.9 and Afghanistan 35.9 \pm 19.1 . Mean assistance at birth by skilled professional and CS rates was $57.25 \pm 27.7$ and $16.8 \pm 20.3$ respectively for 128 provinces (including Egypt). CS delivery was highest in Egypt 51.96 \pm 13.3 and lowest in Afghanistan 2.1 \pm 1.9 , Somalia 2.4 \pm 1.8 and Yemen $4.9 \pm 3.3$ this coincided with the percent of skilled birth attendant at birth. Mean NMR and IMR per 1000 live births in the 129 provinces were $24.9 \pm 12.1$ and 48.2 \pm 26.5 respectively. NMR was highest in Somalia (40.8 \pm 16.6$)$, Pakistan

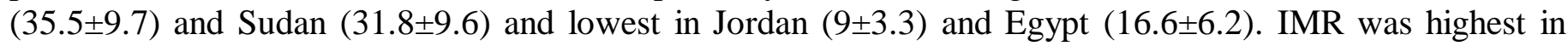
Somalia (69 \pm 19.9$)$, Pakistan 54.5 \pm 13.9 and Sudan $(51.7 \pm 16.1)$ and lowest in Jordan (14.2 \pm 4.2$)$ and Egypt (26.2 \pm 9.2$)$. There were apparant differences between LIC (63) including Afghanistan (33), Somalia (8) and Yemen (22), MIC (55) including Egypt (25), Sudan (18) and Pakistan (12) and HIC (12) except prelacteals and $\mathrm{EBF}$ in first hour.

Table (2) examines the correlations between ANC, delivery by SBA, CS with BFI and NMR, IMR U5MR at country level in seven countries. CS was inversely correlated with BFI $<24$ hours in Afghanistan ( $\mathrm{r}-0.3)$, Jordan (r-0.5), and Pakistan. It was positively correlated with BFI $<24$ hours in Sudan (r0.7), but not correlated with BFI in Somalia and Yemen $(\mathrm{P}>0.05)$. CS correlated with intake of prelacteals in Sudan ( $\mathrm{r} 0.7)$ and Yemen (r0.5) and inversely in Afghanistan (r-0.3) but not in the other countries. CS correlated inversely with NMR, IMR and U5 MR in Egypt and Sudan but positively in Jordan but not or insignificantly so in other countries.

Delivery by SBA was not or inversely associated with EIBF in almost all the countries but ANC was negatively associated with EIBF in Afghanistan, Jordan and Pakistan but not in the remaining countries as shown in Table (2).

Table (3) presents the correlations between CS and other variables for all the 128 provinces for the seven countries under study. shows that CS was strongly associated with adequate ANC ( $>4$ times) and delivery by SBA and inversely correlated with NMR, IMR and U5MR at $\mathrm{P}<0.0001$. ANC was associated with delivery by SBA and inversely correlated with mortality rates in early childhood at $\mathrm{P}<0.0001$. Delivery by SBA was also strongly inversely correlated with NMR, IMR and U5MR in all the provinces of the seven countries under study irrespective of Cesarean rates.

Overall CS correlated inversely with mortality rates IMR (r-0.5), NMR (r-0.4) and U5MR (r-0.5) P<0.05 as shown in table (4). However table (5) demonstrates that the effect of CS on mortality vaired when cut-offs of CS rates were used. It presents the actual relationship between various cut offs of CS rates and BFI in first hour and first day, prelacteals given, delivery by skilled birth attendant, neonatal, infant and under-five mortality in the provinces of the 7 countries of the EMR.

CS cut off below $6 \%$ correlated significantly with EIBF in first hour $(\mathrm{r} 0.6, \mathrm{P}<0.01)$ and delivery by SBA (r0.3, P<0.01) but not with NMR, IMR or U5MR. CS cut off $6-17 \%$ was inversely correlated with BFI within 24 hours of birth $(\mathrm{r} 0.8, \mathrm{P}<0.01)$ and delivery by SBA $(\mathrm{r}-0.7, \mathrm{P}<0.01)$ and positively with intake of 
prelacteals $(\mathrm{r} 0.65, \mathrm{P}<0.01)$ but not with mortality rates. CS cut off $>18 \%$ to $38 \%$ correlated inversely with ANC $>4$ times $(\mathrm{r}-0.7, \mathrm{P}<0.01)$, delivery by SBA $(\mathrm{r}-0.9, \mathrm{P}<0.01)$ and intake of prelacteals $(\mathrm{r}-0.6, \mathrm{P}<0.01)$ but positively with IMR $(\mathrm{r} 0.5, \mathrm{P}<0.01)$ and U5MR $(\mathrm{r} 0.6, \mathrm{P}<0.01)$. CS above $40 \%$ were only reported in Egypt where EIBF at provincial level was not available but correlations between CS with ANC $(\mathrm{r} 0.8, \mathrm{P}<0.01)$ and SBA were significant $(0.6$ at $\mathrm{P}<0.01)$ and inversely with IMR $(\mathrm{r}-0.5)$ but not with NMR or U5MR $(\mathrm{P}>0.05)$.

Table (6) presents CS rates, number of unnecessary CS for the year 2008 cumulative cost in 13 countries of EMR and how this correlated with mortality rates in children, healthy life expectancy and early breastfeeding initiation. CS correlated with healthy life expectancy (r0.5) and inversely with NMR (r-0.2), IMR ( $\mathrm{r}-0.2)$ and U5MR ( $\mathrm{r}-0.4)$ but it was not statistically significant $(\mathrm{P}>0.05)$. Percent unnecessary CS correlated inversely with healthy life expectancy (r-0.4) but positively with NMR (r0.6), IMR (r0.6) and U5MR (r0.6) at $\mathrm{P}<0.05$. Unnecessary CS, NMR, IMR and U5MR were highly correlated inversely with cumulative cost of $\mathrm{CS}$ at $\mathrm{P}<0.01$. EIBF in first hour of birth was not correlated CS or percent unnecessary CS, but EIBF was not correlated with NMR (0.45) and IMR (0.37) and U5MR ( $r-0.4) \mathrm{P}>0.05$. The findings were not conclusive as the numbers were too small.

Figure (1) presents the type of birth attendant in each of the seven countries. In countries like Egypt and Jordan the physician was the predominant birth attendant. While in countries as Afghanistan, Pakistan, Somalia and Yemen the nurses, midwives and traditional birth attendants took the upper hand in delivery. In Sudan the predominant SBA were certified midwives. Mean CS delivery rate for each country was plotted against the SBA and was found to increase when majority of SBA were physicians.

Figure (2) shows that by socioeconomic status mortality rates and CS decreased by increasing wealth quintile, while EIBF and intake of prelacteals did not change much by level of wealth.

Table (6) and figure (3) compare the various variables under study in relation to level of education of the mothers in the provinces of the seven countries under study. There were significant decreases in ANC, CS and SBA and increases in NMR, IMR, U5MR in the poorer social classes. However early EIBF, SSC and prelacteals given at birth were not influenced by level of education.

Table (1) Comparison of mean rates of breastfeeding initiation, cesarean-section delivery, prelacteal feeding practices and mortality rates in 7 EMR countries at provincial level and by income country groups (low, middle and high income groups)

\begin{tabular}{|c|c|c|c|c|c|c|c|c|c|}
\hline $\begin{array}{c}\text { Country } \\
\text { (\# provinces) }\end{array}$ & $\begin{array}{l}\text { EIBF } \\
\text { 1st hr }\end{array}$ & $\begin{array}{c}\text { EIBF } \\
<24 \mathrm{hrs}\end{array}$ & $\begin{array}{c}\text { Prelacteal } \\
\text { feeds offered }\end{array}$ & $\begin{array}{c}\mathrm{ANC}>4 \\
\text { times }\end{array}$ & $\begin{array}{l}\text { delivery by } \\
\text { SBA }\end{array}$ & $\begin{array}{l}\text { Delivery } \\
\text { by CS }\end{array}$ & $\begin{array}{c}\text { NMR/ } \\
1000 \text { LB }\end{array}$ & $\begin{array}{c}\text { IMR/r } \\
1000 \text { LB }\end{array}$ & $\begin{array}{l}\text { U5MR/ } \\
1000 \mathrm{LB}\end{array}$ \\
\hline $\begin{array}{l}\text { Afghanistan } \\
\text { (33) }\end{array}$ & $\begin{array}{l}46.02 \\
\pm 18.2 \\
\end{array}$ & $\begin{array}{l}91.7 \\
\pm 7.3 \\
\end{array}$ & $\begin{array}{c}35.9 \\
\pm 19.4 \\
\end{array}$ & \begin{tabular}{|l}
56.65 \\
\pm 22.02 \\
\end{tabular} & $\begin{array}{l}46.85 \\
\pm 20.8 \\
\end{array}$ & $\begin{array}{c}2.1 \\
\pm 1.9 \\
\end{array}$ & $\begin{array}{c}26.0 \\
\pm 12.6 \\
\end{array}$ & $\begin{array}{r}48.6 \\
\pm 23.8 \\
\end{array}$ & $\begin{array}{c}58.6 \\
\pm 30.86 \\
\end{array}$ \\
\hline Egypt (25) & & & & $\begin{array}{l}83.29 \\
\pm 8.99 \\
\end{array}$ & $\begin{array}{c}92.5 \\
\pm 7.33 \\
\end{array}$ & $\begin{array}{c}51.9 \\
\pm 13.6 \\
\end{array}$ & $\begin{array}{l}16.36 \\
\pm 6.5\end{array}$ & $\begin{array}{l}25.36 \\
\pm 9.66 \\
\end{array}$ & $\begin{array}{c}25.6 \\
\pm 19.3 \\
\end{array}$ \\
\hline Jordan (12) & $\begin{array}{l}63.9 \\
\pm 8.1\end{array}$ & $\begin{array}{l}80.5 \\
\pm 5.4\end{array}$ & $\begin{array}{r}40.4 \\
\pm 5.5\end{array}$ & $\begin{array}{r}92.2 \\
\pm 3.08\end{array}$ & $\begin{array}{r}99.7 \\
\pm 0.24\end{array}$ & $\begin{array}{l}25.1 \\
\pm 5.1\end{array}$ & $\begin{array}{c}9 \\
\pm 3.4\end{array}$ & $\begin{array}{l}14.2 \\
\pm 4.4\end{array}$ & $\begin{array}{l}16.5 \\
\pm 4.1\end{array}$ \\
\hline Pakistan (12) & $\begin{array}{l}30.3 * * \\
\pm 16.6 \\
\end{array}$ & $\begin{array}{c}69.5 \\
\pm 16.8 \\
\end{array}$ & $\begin{array}{c}65.3 \\
\pm 21.4 \\
\end{array}$ & $\begin{array}{r}82.8 \\
\pm 13.9 \\
\end{array}$ & $\begin{array}{c}68.1 \\
\pm 17.2 \\
\end{array}$ & $\begin{array}{l}18.02 \\
\pm 12.9\end{array}$ & $\begin{array}{c}35.5 \\
\pm 10.1\end{array}$ & $\begin{array}{c}54.1 \\
\pm 14.5\end{array}$ & $\begin{array}{c}-63.8 \\
\pm 19.3\end{array}$ \\
\hline Somalia $(8) *$ & $\begin{array}{c}61.5 \\
\pm 5.01\end{array}$ & $\begin{array}{l}86.1 \\
\pm 3.4\end{array}$ & $\begin{array}{c}44.86 \\
\pm 4.8\end{array}$ & $\begin{array}{l}12.56 \\
\pm 5.7\end{array}$ & $\begin{array}{l}38.09 \\
\pm 12.8\end{array}$ & $\begin{array}{c}2.8 \\
\pm 2.4\end{array}$ & $\begin{array}{l}40.8 \\
\pm 9.8\end{array}$ & $\begin{array}{c}69 \\
\pm 18.6\end{array}$ & $\begin{array}{r}89.2 \\
\pm 223\end{array}$ \\
\hline Sudan (18) & $\begin{array}{c}67.6 \\
\pm 12.4\end{array}$ & $\begin{array}{r}87.1 \\
\pm 5.8\end{array}$ & $\begin{array}{c}27.4 \\
\pm 12.2\end{array}$ & $\begin{array}{r}50.4 \\
\pm 11.9 \\
\end{array}$ & $\begin{array}{r}75.8 \\
\pm 18.5 \\
\end{array}$ & $\begin{array}{c}6.2 \\
\pm 5.9 \\
\end{array}$ & $\begin{array}{r}31.8 \\
\pm 9.9 \\
\end{array}$ & $\begin{array}{c}51.7 \\
\pm 16.5 \\
\end{array}$ & $\begin{array}{c}69.5 \\
\pm 22.4 \\
\end{array}$ \\
\hline Yemen (20) & $\begin{array}{c}55.1 \\
\pm 10.1 \\
\end{array}$ & $\begin{array}{l}78.7 \\
\pm 8.7 \\
\end{array}$ & $\begin{array}{c}65.5 \\
\pm 12.8 \\
\end{array}$ & $\begin{array}{l}25.45 \\
\pm 12.4 \\
\end{array}$ & $\begin{array}{c}47.4 \\
\pm 18.8 \\
\end{array}$ & $\begin{array}{c}4.9 \\
\pm 3.4 \\
\end{array}$ & $\begin{array}{r}25.9 \\
\pm 5.5 \\
\end{array}$ & $\begin{array}{c}44.6 \\
\pm 11.9 \\
\end{array}$ & $\begin{array}{c}54.1 \\
\pm 14.8 \\
\end{array}$ \\
\hline & $\begin{array}{l}\text { EIBF } \\
\text { 1st hr }\end{array}$ & $\begin{array}{c}\text { EIBF } \\
<24 \mathrm{hrs}\end{array}$ & $\begin{array}{l}\text { Prelacteal } \\
\text { feeds }\end{array}$ & $\begin{array}{c}\mathrm{ANC}>4 \\
\text { times }\end{array}$ & $\begin{array}{c}\text { delivery by } \\
\text { SBA }\end{array}$ & CS & $\begin{array}{c}\text { NMR/ } \\
1000 \text { LB }\end{array}$ & $\begin{array}{c}\text { IMR/r } \\
1000 \text { LB }\end{array}$ & $\begin{array}{l}\text { U5MR/ } \\
1000 \text { LB }\end{array}$ \\
\hline LIC (59) & $\begin{array}{c}50.6 \\
\pm 15.8\end{array}$ & $\begin{array}{l}86.6 \\
\pm 9.7\end{array}$ & $\begin{array}{c}47.2 \\
\pm 21.3\end{array}$ & $\begin{array}{c}41.8 \\
\pm 24.9\end{array}$ & $\begin{array}{c}46.3 \\
\pm 19.5\end{array}$ & $\begin{array}{c}3.2 \\
\pm 2.9\end{array}$ & $\begin{array}{r}27.3 \\
\pm 11.1\end{array}$ & $\begin{array}{c}48.9 \\
\pm 20.8\end{array}$ & $\begin{array}{c}59.6 \\
\pm 26.9\end{array}$ \\
\hline $\operatorname{MIC}(56)^{2}$ & $\begin{array}{c}51.9 \\
\pm 23.4\end{array}$ & $\begin{array}{r}79.7 \\
\pm 14.5 \\
\end{array}$ & $\begin{array}{r}43.3 \\
\pm 25.1 \\
\end{array}$ & $\begin{array}{r}72.6 \\
\pm 18.9 \\
\end{array}$ & $\begin{array}{c}81.5 \\
\pm 17.4\end{array}$ & $\begin{array}{c}29.4 \\
\pm 23.7\end{array}$ & $\begin{array}{r}25.8 \\
\pm 12.1 \\
\end{array}$ & $\begin{array}{r}40.5 \\
\pm 18.9\end{array}$ & $\begin{array}{r}48.6 \\
\pm 28.9\end{array}$ \\
\hline HIC (12) & $\begin{array}{l}63.9 \\
\pm 8.2\end{array}$ & $\begin{array}{r}80.5 \\
\pm 5.4\end{array}$ & $\begin{array}{l}40.4 \\
\pm 5.5\end{array}$ & $\begin{array}{l}92.2 \\
\pm 3.1\end{array}$ & $\begin{array}{c}99.7 \\
\pm 0.25\end{array}$ & $\begin{array}{l}25.1 \\
\pm 5.1\end{array}$ & $\begin{array}{r}9.0 \\
\pm 3.4\end{array}$ & $\begin{array}{r}14.2 \\
\pm 4.4\end{array}$ & $\begin{array}{l}16.6 \\
\pm 4.1\end{array}$ \\
\hline Total & $\begin{array}{c}52.6 \\
\pm 18.2\end{array}$ & $\begin{array}{c}83.8 \\
\pm 11.4\end{array}$ & $\begin{array}{c}43.8 \\
\pm 22.7\end{array}$ & $\begin{array}{c}60.1 \\
\pm 27.7\end{array}$ & $\begin{array}{c}66.9 \\
\pm 26.5\end{array}$ & $\begin{array}{l}20.4 \\
\pm 1.8\end{array}$ & $\begin{array}{r}24.9 \\
\pm 12.2\end{array}$ & $\begin{array}{r}41.9 \\
\pm 21.3\end{array}$ & $\begin{array}{c}50.7 \\
\pm 29.1\end{array}$ \\
\hline
\end{tabular}

LIC: low income countries and include Afghanistan, Somalia, Sudan and Yemen, MIC: middle income countries and include Pakistan and Egypt, HIC: high income countries include Jordan. Mortality rates were calculated only for 5 provinces in Somaliland. EIBF: Breastfeeding initiation, CS: Cesarean section, NMR: neonatal mortality rate, IMR: infant mortality rate. ${ }^{2}$ Egypt data for EBF and prelacteals excluded (31 cases analyzed instead of 56). 
Table (2) Relationship between early breastfeeding practices and perinatal practices in the 102 provinces of the six regions under study ${ }^{1}$

\begin{tabular}{|c|c|c|c|c|}
\hline & & $\begin{array}{c}\text { Antenatal care visits }>4 \\
\text { (102) }\end{array}$ & $\begin{array}{l}\text { Delivery by SBA } \\
\text { (102) }\end{array}$ & $\begin{array}{c}\text { Cesarean section } \\
\text { (102) }\end{array}$ \\
\hline \multirow{2}{*}{$\begin{array}{l}\text { Initiation of breastfeeding in the first } \\
\text { hour of birth }\end{array}$} & Pearson Correlation & $\begin{array}{l}\mathrm{r} 0.147 \\
\end{array}$ & $\mathrm{r} 0.24 *$ & $\mathrm{r}-0.2$ \\
\hline & Sig. (2-tailed) & 0.141 & 0.015 & 0.1 \\
\hline \multirow{2}{*}{$\begin{array}{l}\text { Initiation of breastfeeding in the first } \\
24 \text { hour of birth }\end{array}$} & Pearson Correlation & $\mathrm{r}-0.129$ & $\mathrm{r} 0.25^{*}$ & $\mathrm{r} 0.2$ \\
\hline & Sig. (2-tailed) & 0.197 & 0.012 & 0.06 \\
\hline \multirow{2}{*}{ Prelacteals given at birth } & Pearson Correlation & $\mathrm{r} 0.018$ & $\mathrm{r}-0.4 * *$ & $\mathrm{r}-0.3 * *$ \\
\hline & Sig. (2-tailed) & 0.858 & 0.000 & 0.008 \\
\hline
\end{tabular}

**. Correlation is significant at the 0.01 level (2-tailed). *Correlation is significant at the 0.05 level (2-tailed). 1Data exclude Egypt governorates

Table (3) Correlations between antenatal care, delivery by skilled health professional, delivery by cesarean section with, breastfeeding initiation and neonatal, infant and under-five mortality rates

\begin{tabular}{|c|c|c|c|c|c|c|}
\hline (2) & $\begin{array}{c}\text { EIBF 1st } \\
\text { hr }\end{array}$ & $\begin{array}{c}\text { EIBF }<24 \\
\text { hr } \\
\end{array}$ & $\begin{array}{c}\text { Prelacteals } \\
\text { given }\end{array}$ & NMR & IMR & U5MR \\
\hline $\begin{array}{c}\text { Afghanistan (33) } \\
\text { Cesarean section } \\
\text { Delivery by SBA } \\
\text { ANC >4 times }\end{array}$ & $\begin{array}{c}\mathbf{r 0 . 3} \\
\mathbf{r}-0.2 \\
\mathbf{r}-0.07 \\
\end{array}$ & $\begin{array}{c}\mathbf{r}-0.3 \\
\mathbf{r}-0.1 \\
\mathbf{r - 0 . 0 0 1}\end{array}$ & $\begin{array}{c}\text { r-0.14 } \\
\text { r-0.2 } \\
\text { r0.2 } \\
\end{array}$ & $\begin{array}{c}\mathrm{r}-0.1 \\
\mathrm{r}-0.3 \\
\mathrm{r}-0.06 \\
\end{array}$ & $\begin{array}{c}\mathbf{r}-0.15 \\
\mathbf{r}-0.4 \\
\mathrm{r}-0.1 \\
\end{array}$ & $\begin{array}{c}\mathbf{r}-0.14 \\
\mathbf{r}-0.5 \\
\mathbf{r}-0.2 \\
\end{array}$ \\
\hline $\begin{array}{c}\text { Egypt (25) } \\
\text { Cesarean section } \\
\text { Delivery by SBA } \\
\text { ANC }>4 \text { times } \\
\end{array}$ & & & & $\begin{array}{c}\mathbf{r}-0.4 \\
\mathrm{r}-0.26 \\
\mathrm{r}-0.3\end{array}$ & $\begin{array}{c}\mathbf{r}-0.5 * \\
\mathbf{r}-0.4 \\
\mathbf{r}-0.43 *\end{array}$ & $\begin{array}{c}-\mathrm{r} 0.25 \\
\mathrm{r}-0.4 \\
\mathrm{r}-0.4^{*}\end{array}$ \\
\hline $\begin{array}{l}\text { Jordan (12) } \\
\text { Cesarean section } \\
\text { Delivery by SBA } \\
\text { ANC >4 times } \\
\text { STS after birth } \\
\end{array}$ & $\begin{array}{l}\mathrm{r}-0.1 \\
\mathrm{r}-0.4 \\
\mathrm{r}-0.5 \\
\mathrm{r} 0.9^{* *}\end{array}$ & $\begin{array}{l}\mathrm{r}-0.5 \\
\mathrm{r}-0.17 \\
\mathrm{r}-0.4 \\
\mathrm{r} 0.63^{*}\end{array}$ & $\begin{array}{l}\mathrm{r} 0.2 \\
\mathrm{r} 0.15 \\
\mathrm{r} 0.4 \\
\mathrm{r}-0.15\end{array}$ & $\begin{array}{l}\mathrm{r} 0.6 \\
\mathrm{r}-0.04 \\
\mathrm{r} 0.11 \\
\mathrm{r} 0.13\end{array}$ & $\begin{array}{l}\mathrm{r} 0.6 \\
\mathrm{r}-0.3 \\
\mathrm{r} 0.07 \\
\mathrm{r} 0.18\end{array}$ & $\begin{array}{l}\mathrm{r} 0.5 \\
\mathrm{r}-0.4 \\
\mathrm{r} 0.16 \\
\mathrm{r} 0.4\end{array}$ \\
\hline $\begin{array}{l}\text { Pakistan (12) } \\
\text { Cesarean section } \\
\text { Delivery by SBA } \\
\text { Any ANC } \\
\text { STS after birth }\end{array}$ & $\begin{array}{l}r-0.4 \\
r-0.5 \\
r-0.7^{*} \\
r-0.5^{*}\end{array}$ & $\begin{array}{l}r-0.45 \\
r-0.3 \\
r 0.5 \\
r-0.3\end{array}$ & $\begin{array}{l}\mathrm{r} 0.16 \\
\mathrm{r} 0.1 \\
0.24 \\
\mathrm{r}-0.34\end{array}$ & $\begin{array}{l}\mathrm{r} 0.15 \\
\mathrm{r} 0.003 \\
\mathrm{r} 0.04 \\
\mathrm{r}-0.15\end{array}$ & $\begin{array}{l}\mathrm{r}-0.02 \\
\mathrm{r} 0.3 \\
\mathrm{r}-0.3 \\
\mathrm{r}-0.3\end{array}$ & $\begin{array}{l}\mathrm{r}-0.1 \\
\mathrm{r}-0.4 \\
\mathrm{r}-0.33 \\
\mathrm{r}-0.3\end{array}$ \\
\hline $\begin{array}{l}\text { Somalia (8) } \\
\text { Cesarean section } \\
\text { Delivery by SBA } \\
\text { ANC >4 times }(1)\end{array}$ & $\begin{array}{l}\mathrm{r} 0.2 \\
\mathrm{r} 0.4 \\
\mathrm{r} 0.3\end{array}$ & $\begin{array}{l}\mathrm{r}-0.3 \\
\mathrm{r} 0.17 \\
\mathrm{r}-0.04\end{array}$ & $\begin{array}{l}r-0.3 \\
r-0.3 \\
r-0.4\end{array}$ & $\begin{array}{l}\mathrm{r} 0.24 \\
\mathrm{r} 0.12 \\
\mathrm{r}-0.03\end{array}$ & $\begin{array}{l}\mathrm{r} 0.14 \\
\mathrm{r} 0.005 \\
\mathrm{r} 0.02\end{array}$ & $\begin{array}{l}\mathrm{r} 0.11 \\
\mathrm{r} 0.3 \\
\mathrm{r} 0.3\end{array}$ \\
\hline $\begin{array}{l}\text { Sudan (18) } \\
\text { Cesarean section } \\
\text { Delivery by SBA } \\
\text { ANC >4 times }\end{array}$ & $\begin{array}{l}\mathrm{r} 0.54 \\
\mathrm{r} 0.3 \\
\mathrm{r}-0.04 \\
\end{array}$ & $\begin{array}{l}\mathrm{r} 0.7 \\
\mathrm{r} 0.2 \\
\mathrm{r} 0.4\end{array}$ & $\begin{array}{l}\mathrm{r} 0.6 * * \\
\mathrm{r} 0.001 \\
\mathrm{r} 0.4\end{array}$ & $\begin{array}{l}\mathrm{r}-0.43 \\
\mathrm{r}-0.11 \\
\mathrm{r}-0.3\end{array}$ & $\begin{array}{l}r-0.6 * * \\
r-0.03 \\
r-0.3\end{array}$ & $\begin{array}{l}\mathrm{r} 0.77 * * \\
\mathrm{r}-0.001 \\
\mathrm{r}-0.4\end{array}$ \\
\hline $\begin{array}{l}\text { Yemen (20) } \\
\text { CS } \\
\text { Delivery by SBA } \\
\text { Any ANC }\end{array}$ & $\begin{array}{l}\mathrm{r} 0.12 \\
\mathrm{r} 0.002 \\
\mathrm{r} 0.2\end{array}$ & $\begin{array}{l}\mathrm{r} 0.04 \\
\mathrm{r} 0.07 \\
\mathrm{r} 0.01\end{array}$ & $\begin{array}{l}\mathrm{r} 0.5 \\
\mathrm{r} 0.6^{* *} \\
\mathrm{r} 0.5^{*}\end{array}$ & $\begin{array}{l}\mathrm{r}-0.3 \\
\mathrm{r}-0.4 \\
\mathrm{r}-0.3\end{array}$ & $\begin{array}{l}\mathrm{r}-0.3 \\
\mathrm{r}-0.5^{*} \\
\mathrm{r}-0.5^{*}\end{array}$ & $\begin{array}{l}r-0.4 \\
r-0.54 * \\
r-0.5 *\end{array}$ \\
\hline
\end{tabular}

$\mathrm{P}<0.05^{*}, \mathrm{P}<0.01^{* *}$, ANC: antenatal care, SBA: skilled birth attendant (1) data for ANC only available for North East (3 provinces) and not

Somaliland (5 provinces). 2. No data for Egypt governorates were available for EIBF and feeding prelacteals in secondary analysis.

Table (4) Relationship between Cesarean section and perinatal practices with mortality in children of the 129 provinces of the six countries under study

\begin{tabular}{|c|c|c|c|c|c|c|c|}
\hline & & CS (129) & $\begin{array}{c}\text { ANC4 } \\
(129)\end{array}$ & SBA (129) & NMR (129) & IMR (129) & $\begin{array}{c}\text { U4MR } \\
(129)\end{array}$ \\
\hline \multirow{2}{*}{$\begin{array}{l}\text { Delivery by C- } \\
\text { Section }\end{array}$} & \begin{tabular}{|l} 
Pearson \\
Correlation
\end{tabular} & 1 & $\mathrm{r} 0.620 * *$ & $\mathrm{r} 0.702 * *$ & $\mathrm{r}-0.423 * *$ & $\mathrm{r}-0.507 * *$ & $\mathrm{r}-0.541 * *$ \\
\hline & Sig. (2-tailed) & & .000 & .000 & .000 & .000 & .000 \\
\hline \multirow{2}{*}{$\begin{array}{l}\text { Antenatal } \\
\text { care }>4 \text { times }\end{array}$} & $\begin{array}{l}\text { Pearson } \\
\text { Correlation }\end{array}$ & $\mathrm{r} 0.620 * *$ & 1 & $\mathrm{r} 0.654 * *$ & $\mathrm{r}-0.345^{* *}$ & $\mathrm{r}-0.407 * *$ & $\mathrm{r}-0.457 * *$ \\
\hline & Sig. (2-tailed) & .000 & & .000 & .000 & .000 & .000 \\
\hline \multirow{2}{*}{$\begin{array}{l}\text { Delivery by } \\
\text { skilled birth } \\
\text { attendant }\end{array}$} & $\begin{array}{l}\text { Pearson } \\
\text { Correlation }\end{array}$ & $\mathrm{r} 0.702 * *$ & $\mathrm{r} 0.654 * *$ & 1 & $\mathrm{r}-0.462 * *$ & $\mathrm{r}-0.597 * *$ & $\mathrm{r}-0.605 * *$ \\
\hline & Sig. (2-tailed) & .000 & .000 & & .000 & .000 & .000 \\
\hline
\end{tabular}

**. Correlation is significant at the 0.01 level (2-tailed). 


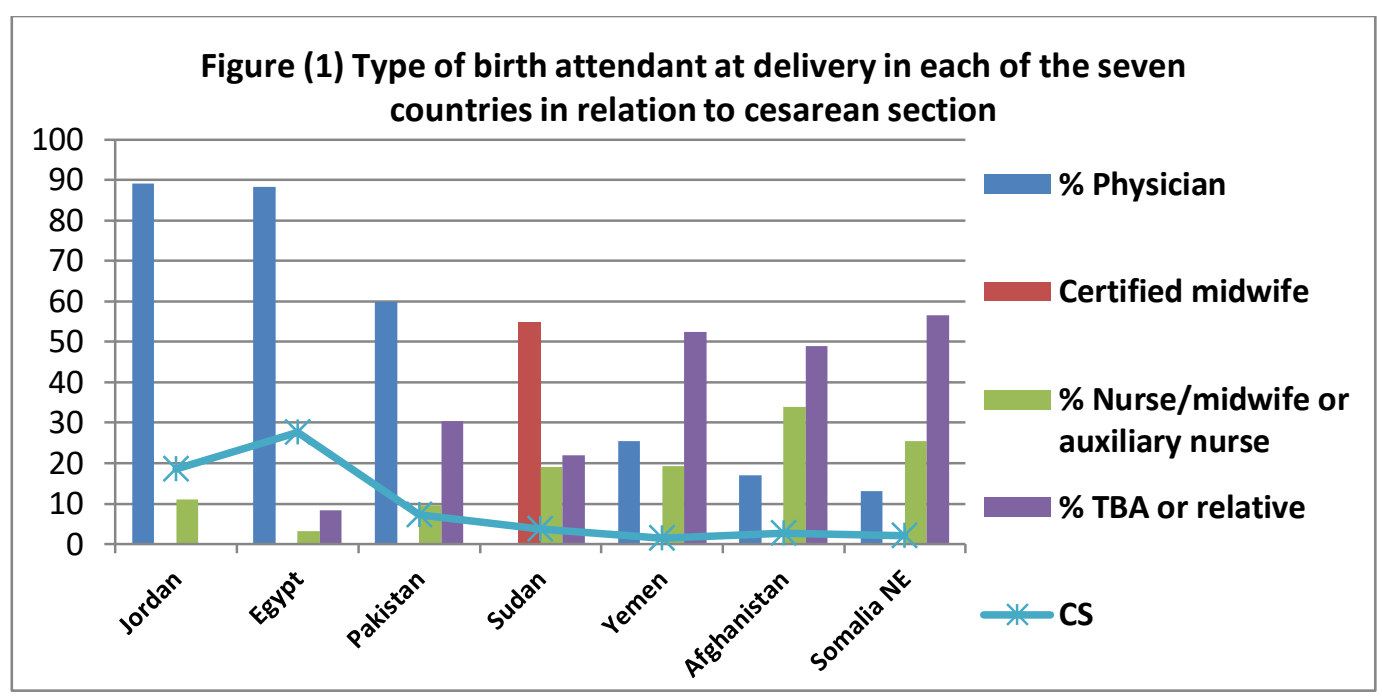

Table (5) Relationship between cesarean section cut off rates and early breastfeeding initiation, prelacteals, skilled birth attendant, neonatal, infant and under-five mortality in the 128 provinces of the 7 countries of the EMR

\begin{tabular}{|l|c|c|c|c|c|c|c|c|}
\hline $\begin{array}{l}\text { Percent CS } \\
\text { delivery rates }\end{array}$ & ANC>4 & $\begin{array}{c}\text { EIBF } \\
\mathbf{1 h r}\end{array}$ & EIBF $<\mathbf{2 4 h r}$ & $\begin{array}{c}\text { Prelacteals } \\
\text { given }\end{array}$ & SBA & NMR & IMR & U5MR \\
\hline $\begin{array}{l}\text { CS <5 } \\
(\mathbf{6 1})\end{array}$ & $\mathrm{r}-0.1$ & $\mathrm{r}-0.6^{* *}$ & $\mathrm{r}-0.2$ & $\mathrm{r} 0.5^{* *}$ & $\mathrm{r}-0.3^{* *}$ & $\mathrm{r}-$ & $\mathrm{r} 0.06$ & $\mathrm{r}-0.06$ \\
\hline $\begin{array}{l}\text { CS 6-17 } \\
(\mathbf{2 3})\end{array}$ & $\mathrm{r}-0.2$ & $\mathrm{r} 0.04$ & $\mathrm{r}-0.8^{* *}$ & $\mathrm{r} 0.65^{* *}$ & $\mathrm{r}-0.7^{* *}$ & $\mathrm{r}-0.2$ & $\mathrm{r} 0.15$ & $\mathrm{r} 0.1$ \\
\hline $\begin{array}{l}\text { CS 18- 38 } \\
(\mathbf{1 7})\end{array}$ & $\mathrm{r}-0.7^{* *}$ & $\mathrm{r}-0.17$ & $\mathrm{r}-0.34$ & $\mathrm{r}-0.6^{* *}$ & $\mathrm{r}-0.9^{* *}$ & $\mathrm{r} 0.34$ & $\mathrm{r} 0.5^{*}$ & $\mathrm{r} 0.6^{* *}$ \\
\hline $\begin{array}{l}\text { CS }>\text { 40* } \\
(\mathbf{2 5})\end{array}$ & $\mathrm{r} 0.8^{* *}$ & & & & $\mathrm{r} 0.7^{* *}$ & $\mathrm{r}-0.4$ & $\mathrm{r}-0.5^{*}$ & $\mathrm{r}-0.25$ \\
\hline
\end{tabular}

** . Correlation is significant at the 0.01 level (2-tailed). ${ }^{*}$ Correlation is significant at the 0.05 level (2-tailed).

Table (6) Cesarean section rates, number of unnecessary cesarean sections for year 2008 in 13 countries of Eastern Mediterranean region cumulative cost and how this correlated with mortality rates in children, healthy life expectancy and breastfeeding initiation

\begin{tabular}{|c|c|c|c|c|c|c|c|c|c|}
\hline \multirow[b]{2}{*}{ Country } & \multirow{2}{*}{$\begin{array}{c}\text { CS } \\
\text { rate } \\
(\%) \\
\end{array}$} & \multicolumn{3}{|c|}{ Unnecessary CS for year 2008} & \multirow[t]{2}{*}{$\begin{array}{l}\text { Healthy life } \\
\text { expectancy }\end{array}$} & \multirow[t]{2}{*}{ NMR } & \multirow[t]{2}{*}{ IMR } & \multirow{2}{*}{$\begin{array}{c}\text { U5MR per } \\
1000 \text { live } \\
\text { births }\end{array}$} & \multirow[t]{2}{*}{ EIBF } \\
\hline & & no & $\%$ & Cumulative cost & & & & & \\
\hline Bahrain & 16.0 & 140 & 0.0 & 100 & 67 & 1 & 5 & 6 & \\
\hline Iran & 41.9 & 373,372 & 6.0 & 71.5 & 66.6 & 18 & 27 & 16 & 68.7 \\
\hline Egypt & 27.6 & 253,890 & 4.1 & 75.6 & 64.1 & 13 & 20 & 24 & 27.1 \\
\hline Jordan & 18.5 & 5,495 & 0.1 & 99.1 & 65 & 10 & 13 & 18 & 67 \\
\hline Lebanon & 23.3 & 5,280 & 0.1 & 99.3 & 65.7 & 5 & 7 & 8 & 41.3 \\
\hline Libya & 7.5 & 3,675 & 0.1 & 99.5 & 63.8 & 7 & 11 & 13 & \\
\hline Morocco & 5.4 & 29,716 & 0.9 & 88.8 & 64.9 & 18 & 24 & 28 & 26.8 \\
\hline Pakistan & 7.3 & 144,099 & 4.5 & 46.9 & 57.8 & 12 & 18 & 81 & 19.6 \\
\hline Qatar & 15.9 & 135 & 0.0 & 100 & 67.7 & 4 & 7 & 7 & 33.5 \\
\hline Sudan & 3.7 & 81,648 & 2.6 & 66.2 & 55.9 & 30 & 48 & 48 & 68.7 \\
\hline Tunisia & 8.0 & 3,380 & 0.1 & 99.6 & 66.7 & 8 & 12 & 14 & 39.9 \\
\hline Oman & 6.6 & 2,074 & 0.1 & 99.8 & 66.7 & 5 & 10 & 12 & 71.1 \\
\hline Yemen & 1.4 & 72,756 & 2.3 & 68.5 & 57.7 & 22 & 34 & 42 & 52.7 \\
\hline \multicolumn{10}{|c|}{ Correlative studies with: } \\
\hline & $\begin{array}{c}\mathrm{CS} \\
\text { rates }\end{array}$ & - & $\begin{array}{c}\text { \% unnecessary } \\
\text { CS }\end{array}$ & Cumulative cost & $\begin{array}{l}\text { Healthy life } \\
\text { expectancy }\end{array}$ & NMR & IMR & U5MR & EIBF \\
\hline $\begin{array}{l}\text { Healthy life } \\
\text { Exp. (13) }\end{array}$ & $\mathrm{r} 0.5$ & - & $\mathrm{r}-0.42$ & $\mathrm{r} 0.78 * *$ & & - & - & - & $\mathrm{r} 0.00$ \\
\hline NMR (13) & $\mathrm{r}-0.2$ & - & $\mathrm{r} 0.57 *$ & $\mathrm{r}-0.69 * *$ & $\mathrm{r}-0.74$ & - & - & - & $\mathrm{r} 0.3$ \\
\hline IMR (13) & $\mathrm{r}-0.2$ & - & $\mathrm{r} 0.56^{*}$ & $\mathrm{r}-0.69 * *$ & $\mathrm{r}-0.76^{* *}$ & - & - & - & 0.3 \\
\hline U5MR (13) & r- -0.4 & - & $\mathrm{r} 0.56^{*}$ & $\mathrm{r}-0.9 * *$ & $\mathrm{r}-0.86^{* *}$ & - & - & - & $\mathrm{r}-0.3$ \\
\hline $\begin{array}{l}\text { EIBF 1st hr } \\
\text { (11) }\end{array}$ & r0.11 & - & $\mathrm{r}-0.05$ & $\mathrm{r} 0.2$ & $\mathrm{r} 0.001$ & $\mathrm{r} 0.45$ & $\mathrm{r} 0.37$ & r0.4 & - \\
\hline
\end{tabular}


Source of data of for unnecessary cesarean and cumulative cost from "WHO, 2010" Pearson correlation, 2-tailed significance: $\mathrm{P}<0.1 *$, $\mathrm{P}<0.001 * *, \mathrm{P}<0.000^{* * *}$. CS: cesarean delivery rate, NMR: neonatal mortality rate, IMR: infant mortality rate, U5MR: under-five mortality rate, EIBF: early breastfeeding initiation

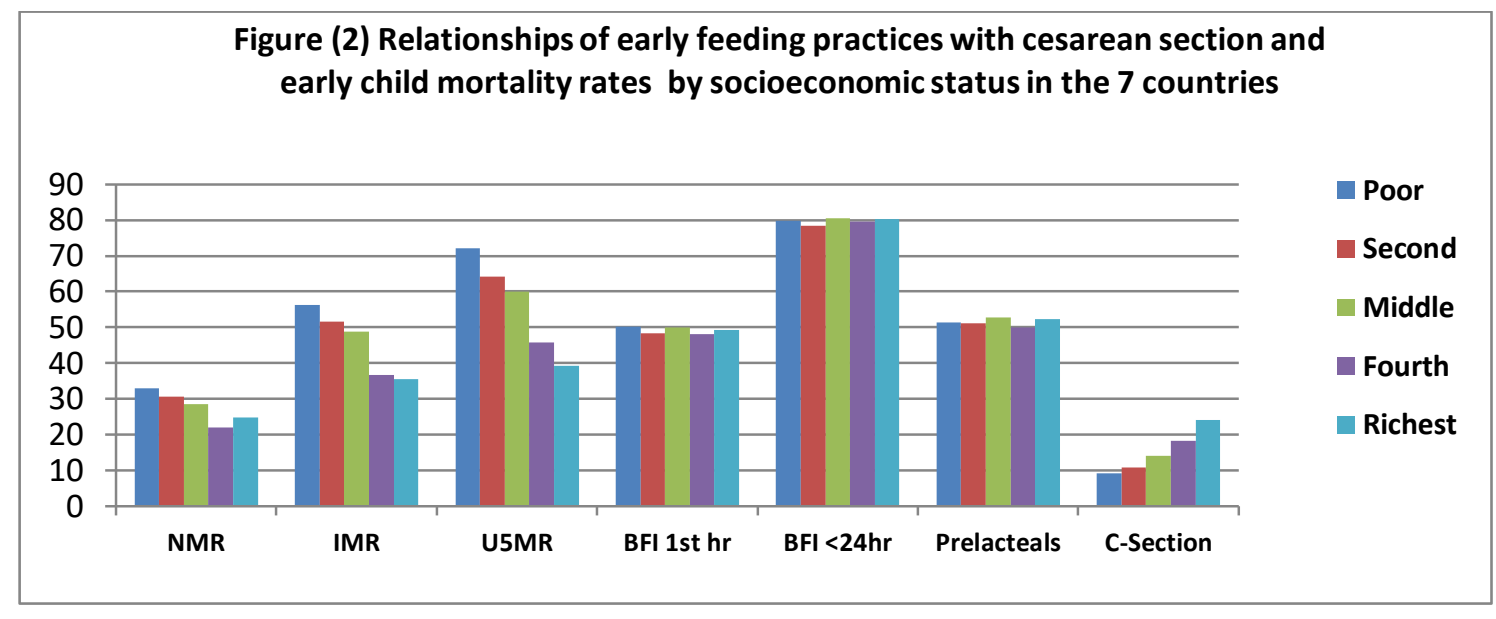

Table (7) Comparison of early feeding practices, delivery practices and early child mortality rates by level of education of mothers in the provinces of the seven EMR countries

\begin{tabular}{|l|l|l|l|l|}
\hline Mean \& SDS for: & No Education & Primary & Secondary* & High \\
\hline EIBF $1^{\text {st }}$ hr of birth & $50.3 \pm 20.1$ & $48.2 \pm 16.7$ & $50.7 \pm 20.9$ & $45.5 \pm 19.9$ \\
\hline EIBF <24hr of birth & $80.2 \pm 10.2$ & $78.2 \pm 10.1$ & $79.8 \pm 12.4$ & $78.7 \pm 14.6$ \\
\hline Prelacteals given & $48.9 \pm 16.5$ & $50.7 \pm 17.9$ & $48.5 \pm 19.7$ & $55.9 \pm 16.6$ \\
\hline No ANC & $32.8 \pm 18.8$ & $19.1 \pm 13.03$ & $10.1 \pm 6.6$ & $2.4 \pm 2.4$ \\
\hline SBA & $57.6 \pm 21.4$ & $76.4 \pm 14.0$ & $87.01 \pm 9.4$ & $95.7 \pm 3.5$ \\
\hline C-Section & $12.3 \pm 13.1$ & $15.8 \pm 13.1$ & $20.8 \pm 13.8$ & $32.4 \pm 16.3$ \\
\hline STS** & 72.2 & 66.4 & 68 & 68.3 \\
\hline NMR & $64.3 \pm 23.8$ & $58.1 \pm 22.8$ & $45.0 \pm 16.4$ & $27.7 \pm 9.2$ \\
\hline IMR & $51.3 \pm 17.8$ & $48.6 \pm 18.75$ & $38.3 \pm 12.4$ & $24.9 \pm 9.4$ \\
\hline U5MR & $30.4 \pm 11.8$ & $28.6 \pm 12.2$ & $25.2 \pm 9.6$ & $16.9 \pm 7.06$ \\
\hline
\end{tabular}

EIBF: breastfeeding initiation, ANC: antenatal care, SBA: skilled birth attendant, C-section: cesarean section delivery, SSC: skin to skin care at birth, NMR: neonatal mortality rate per 1000 live birth, IMR: infant mortality rate per 1000 live birth, U5MR: Under-five mortality rate per 1000 live birth. Secondary also includes middle in a country as Pakistan* Skin to skin contact (SSC) at birth was reported only by Demographic survey in Jordan (2018).

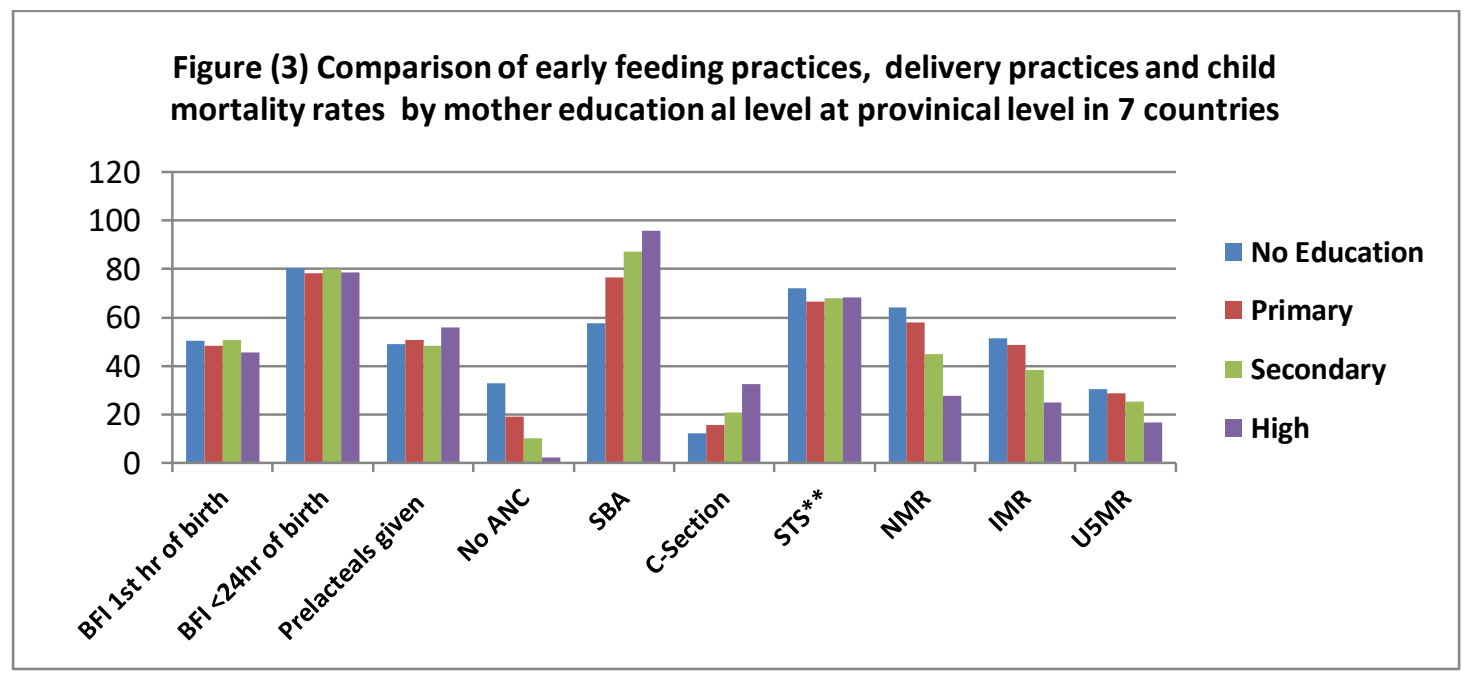

\section{Discussion}

In this study we have used data from demographic health surveys (DHS). The DHS programme represents the largest worldwide effort to obtain nationally representative demographic and health data from household surveys in developing countries. Surveys are implemented by institutions in the host country, usually government statistical offices, and 5,000-30,000 women of childbearing age are interviewed in a standard survey. As the DHS use standardized questionnaires and methods of training, data collection and processing, they are often considered the 'best available gold standard' for many health indicators in developing 
countries and are used for global monitoring efforts ${ }^{(21)}$. DHS figures are considered valid estimations of actual CS rates at country level, although they might be imprecise ${ }^{(22)}$.

The mean CS was lowest in countries with low income as Afghanistan, Somalia and Yemen. It was highest in countries of high socioeconomic levels as in Jordan and lowest in Low income countries as Afghanistan and Yemen. CS varies in lower middle income countries, being very high in Egypt and moderately high with considerable variation between provinces in Pakistan and Sudan as per the World Bank classification. Betran et al. ${ }^{(10)}$ also reported marked variations with CS births being $21.1 \%$ in developed countries, versus only $2 \%$ in developing countries with a worldwide average estimate of around 15\%. In Latin America and the Caribbean it was highest at $29.2 \%$, and in Africa it was lowest at $3.5 \%{ }^{(10)}$.

Our data showed that CS below 6\% was somewhat inversely associated with IMR but poorly with NMR and U5MR. Although values from $6 \%$ to $17 \%$ were also inversely associated with IMR, it was not statistically significant. CS rates of 18 to 38 percent were positively associated with increased NMR, IMR and U5MR. CS over $40 \%$ was strongly associated with presence of SBA and CS but with no association with mortality. This shows that although the overall rates of CS appeared to be protective against child mortality, yet very high and very low had no effect. Moreover the cut offs in CS over the recommended by WHO demonstrate that CS there is a positive correlation with child mortality.

Also our analysis revealed a strong association between unnecessary CS and mortality rates in children and inverse correlations with healthy life expectancy in 13 countries of the EMR. The cumulative cost of unnecessary CS was inversely correlated with mortality and positively with life expectancy, this means that cost reduced from unnecessary CS by early mortality is significantly increased by health problems caused by CS later in life. Our findings are supported by those of Betran et al $(2007)^{(10)}$ and Athabe et al (2006) ${ }^{(11)}$ who showed that in developing countries with low CS there is a strong inverse association between CS rates and maternal, infant and neonatal mortality suggesting that CS when indicated can reduce mortality. However they reported no association was observed in medium- and high-income countries for either neonatal mortality or maternal mortality ${ }^{(10,11)}$. Moreover CS is linked to gross chronic disorders as asthma, inflammatory bowel disease, diabetes mellitus, rheumatoid arthritis and leukemia probably due to altered microbiome that alters baby's immune system later in life ${ }^{(23)}$. Meta-analysis studies showed that exclusive breastfeeding $(\mathrm{EBF})$ helps maintain micorbiome status and prevent dysbiosis that happens to infants born by $\mathrm{CS}$ and not EBF. The effect extends after six months in EBF ${ }^{(24)}$. Hence promoting early EIBF and EBF from birth can reduce morbidity and mortality from exposure to CS.

Although we did not find direct relationships between EIBF and CS at individual country level, overall there was a direct correlation between CS and EIBF rate in first hour when CS rate was $<6$ and an inverse relationship of EIBF at one hour and one day in regions when CS was between 18 to 38. This was also associated with a positive correlation between CS and NMR and IMR. At country level CS was inversely correlated with EIBF in the first 24 hours of life in Pakistan and Jordan indicating that CS may delay EIBF increasing the negative impact CS may have on child survival as shown by the positive correlation of CS with NMR in the provinces where CS ranged from 18 to 38 percent. Hobbs et al (2016) ${ }^{(1)}$ reported that women who delivered by emergency CS were found to have a higher proportion of breastfeeding difficulties (41\%), and used more resources before $(67 \%)$ and after $(58 \%)$ leaving the hospital, when compared to vaginal delivery or planned CS. Women who delivered with a planned CS were more likely to discontinue breastfeeding before 12 weeks postpartum compared to those who delivered vaginally. In this study we found that in high income countries (HICs) like Jordan when CS was performed before labour pains (i.e. planned CS) it was negatively correlated with EIBF. This was not the case for low income countries (LICs) like Sudan where planned CS and unplanned CS were both correlated with EIBF but also correlated with high intake of prelacteals. Hence the high EIBF, despite high intake of prelacteals was associated with the inverse associations between CS and child mortality (NMR and IMR) as in Sudan and Yemen. Hence CS and EIBF may work in synergy to save live births in LICs.

A review study ${ }^{(25)}$ presented evidence that immediate or early skin-to-skin contact between mother and baby, after a CS may increase early EIBF, decrease time to the first breastfeed, reduce formula 
supplementation in hospital, increase bonding and maternal satisfaction, maintain the temperature of newborns and reduce newborn stress. Similar findings were also reported by research workers in Egypt ${ }^{(5)}$.

EIBF has been shown to be high in low and low middle income countries compared to HICs as Jordan. This can explain these differential findings between countries. Hence it is possible that delayed initiation of breastfeeding and offering prelacteal feeds may be confounding factors in influencing mortality rates associated with CS in such situations. Although Jordan had high EIBF rates, yet almost one half of newborns were offered prelacteals. Moreover antenatal care for over 4 times was inversely related with EIBF and positively correlated with NMR and IMR.

In this analysis EMR countries with highest CS were Iran (41.9\%), Egypt (27.6\%), Lebanon (23.3\%) and Jordan (18.5\%), while those with highest unnecessary CS rates were Iran (6\%), Pakistan (4.5\%) and Egypt (4.1\%). Countries with highest cumulative cost from unnecessary CS were Bahrain, Lebanon, Libya Tunisia and Oman. The estimated cost of unnecessary CS, presented by the World Health Organization in 2010, report that countries of the EMR with CS over 15\% total 209,707, 476 US dollars for the 13 EMR countries abstracted from their study ${ }^{(26)}$. The cost was inflated by healthy life expectancy and inversely related to early child mortality which increased by unnecessary CS.

In this study CS seemed to increase with increased socioeconomic status and level of education suggesting that CS high levels are primarily related to economic status and level of education. This has been shown by other researchers ${ }^{(10)}$. This raises the question of social inequality in the accessibility to CS delivery when needed to save lives vis. increased rates of unnecessary CS in the richer classes ${ }^{(27)}$. Other workers demonstrated that women in poor socioeconomic conditions had less access to CS in comparison with those women with low obstetric risk and high economic status ${ }^{(28)}$ and higher education ${ }^{(29)}$. This paradoxical association of CS delivery with poverty explains why we found different correlations of CS with mortality at different cut-offs of CS. In contradistinction, other workers have shown that the rise in CS deliveries is justified in tertiary and quateriary referral hospitals where complicated cases that need CS exceed the WHO cut off of $15 \%{ }^{(30)}$. Still such rates cannot explain the alarmingly high CS rates in countries as Egypt ${ }^{(14)}$ and China ${ }^{(31)}$ and many developed countries ${ }^{(32)}$.

Poor access to CS was shown in some of the provinces in LICs with very low rates of CS <6 percent, and where mortality rates were high. Lack of access to skilled birth professionals and antenatal care are also possible confounders, as our analysis showed inverse correlations between ANC and SBA with mortality rates in Egypt, and Yemen for, but with SBA and not for ANC in Afghanistan and Sudan and for ANC but not SBA in Jordan. However there were insignificant correlations of SBA and ANC with mortality in Pakistan and Somalia. However SBA and ANC were inversely correlated with EIBF in Pakistan and Jordan and positively correlated with intake of prelacteals in Sudan and Yemen indicating a need for increasing the availability of SBA (midwives and obstetricians) in delivery and Baby-friendly practices in the countries of the region. Al-Jawaldeh and Abul-Fadl ${ }^{(33)}$ have shown progress in making hospitals Baby-friendly in the EMR was slow particularly in the low and low middle income countries being halted by the political instabilities and ongoing conflict in these countries.

Cesarean delivery was influenced by the availability and type of SBA; as it increased where physicians were predominant decreased where midwives particularly certified midwives as in the case of Sudan were the predominant birth attendant. Research has shown that medical professionals tend to favour CS while midwives are more tolerant and supportive of natural childbirth and when trained can identify the high risk cases that need referral for operative delivery ${ }^{(34,35)}$. Many countries in the region lack trained or certified midwives that can carry out normal deliveries. In Egypt with the disappearance of the "Dayas" as traditional birth attendants, most of the deliveries are carried out by medical professionals ${ }^{(36)}$. This explains the increasing CS in Jordan ${ }^{(37)}$ and skyrocketing CS rates in Egypt increasing by 4.7 times in the private sector and 2.7 times in the public sector from 2005 to $2014^{(38)}$.

In conclusion the study shows that delivery by CS has a controversial role in influencing infant mortality ${ }^{(39}$, 40). The socioeconomic status plays an important role especially in middle and low income groups $(41,42)$ whereas unnecessary CS is definitely associated with mortality especially when associated with poorly skilled health professionals and lack of early breastfeeding support. Early and exclusive breastfeeding 
continue to have protective effects against the adverse effects on mortality and morbidity that can result from the increasing rates of CS, especially when unnecessary ${ }^{(43)}$. Caesarean birth is associated with shortand long-term risks that can extend many years beyond the current delivery and affect the health of women, children and future pregnancies and must be geared to the limited medical indications for saving lives without interfering with timely breastfeeding initiation ${ }^{(44)}$. Moreover, high rates of CS are associated with substantial health-care costs. Hence the World health organization (WHO) recommends that health education for women is an essential component of antenatal care, implementation of evidence-based clinical practice guidelines with timely second opinion and collaborative midwifery-obstetrician model of care (i.e. a model of staffing based on care provided primarily by midwives ${ }^{(45)}$. Our study supports WHO recommendations and in particular with regards to training health professionals from the community as midwives and urging medical professionals attending births to encourage natural delivery and provide support for early initiation of breastfeeding through skin-to-skin and early and continued exclusive breastfeeding for the first six months of life, for healing the damage from operative delivery ${ }^{(46)}$. This can have positive effects on the economy of countries in the region and on reducing the burden of morbidity, mortality and chronic disorders from unnecessary CS and not breastfeeding.

\section{References}

[1] Hobbs AJ, Mannion CA, McDonald SW, Brockway M, Tough SC. The impact of caesarean section on breastfeeding initiation, duration and difficulties in the first four months postpartum.BMC Pregnancy Childbirth 2016 Apr 26;16:90. doi: 10.1186/s12884-016-0876-1.

[2] Watt S, Sword W, Sheehan D, Foster G, Thabane L, Krueger P, Landy CK. The effect of delivery method on breastfeeding initiation from the Ontario Mother and Infant Study (TOMIS) III. J Obs Gyn Neonatal Nursing 2012;41(6):728-37.

[3] Chen C, Yan Y, Gao X, Xiang S, He Q, Zeng G, Liu S, Sha T, Li L. Influences of Cesarean Delivery on Breastfeeding Practices and Duration: A Prospective Cohort Study. J Hum Lact 2018; 34(3):526534.

[4] Zanardo V, Pigozzo A, Wainer G, Marchesoni D, Gasparoni A, Di Fabio S, Cavallin F, Giustardi A, Trevisanuto D. Early lactation failure and formula adoption after elective caesarean delivery: cohort study. Archives Disease Childhood Fetal Neonatal Edition 2013;98(1):F37-41.

[5] Soliman DR, Khairy M. Does first hour skin-to-skin and follow-up support improve breastfeeding outcomes in cesarean delivery despite anesthesia? MCFC Eg J. Breastfeeding (EJBF) 2014; 10: 2939.

[6] Gibbons L, Belizán JM, Lauer JA, Betran AP, Merialdi M, Althabe F. The Global Numbers and Costs of Additionally Needed and Unnecessary Caesarean Sections Performed per Year: Overuse as a Barrier to Universal Coverage.

[7] Lau Y, Tha PH, Ho-Lim SST, Wong LY, Lim PI, Citra Nurfarah BZM, Shorey S. An analysis of the effects of intrapartum factors, neonatal characteristics, and skin-to-skin contact on early breastfeeding initiation. Matern Child Nutr 2018 Jan;14(1).

[8] Karlstrom A, Lindgren H, Hildingson I. Maternal and infant outcome after caesarean section without recorded medical indication: findings from a Swedish case-control study. BJOG 2013;120(4):47986.

[9] Hall MH, Bewley S. Maternal mortality and mode of delivery. Lancet, 1999; 354 (9180): 776.

[10] Betrán AP, Merialdi M, Lauer JA, Bing-Shun W, Thomas J, Van Look P, Wagner M. Rates of caesarean section: analysis of global, regional and national estimates. Paediatr Perinat Epidemiol 2007; 21 (2):98-113.

[11] Barros FC, Victora CG, Barros AJ, Santos IS, Albernaz E, Matijasevich A, et al. The challenge of reducing neonatal mortality in middle income countries: findings from three Brazilian birth cohorts in 1982, 1993, and 2004. Lancet 2005; 365 (9462): 847-54. 8).

[12] Bodner K, Wierrani F, Grunberger W, Bodner-Adler B. Influence of the mode of delivery on maternal and neonatal outcomes: a comparison between elective cesarean section and planned vaginal delivery in a low-risk obstetric population. Arch Gynecol Obstet. 2011;283(6):1193-8. 
[13] Central Statistics Organization (CSO), Ministry of Public Health (MoPH), and ICF. 2017. Afghanistan Demographic and Health Survey 2015. Kabul, Afghanistan: Central Statistics Organization.

[14] Ministry of Health and Population [Egypt], El-Zanaty and Associates [Egypt], and ICF International. 2015. Egypt Demographic and Health Survey 2014. Cairo, Egypt and Rockville, Maryland, USA: Ministry of Health and Population and ICF International.

[15] Department of Statistics (DOS) and ICF. 2019. Jordan Population and Family and Health Survey 2017-18. Amman, Jordan, and Rockville, Maryland, USA: DOS and ICF.

[16] National Institute of Population Studies (NIPS) [Pakistan] and ICF. 2019. Pakistan Demographic and Health Survey 2017-18. Islamabad, Pakistan, and Rockville, Maryland, USA: NIPS and ICF.

[17] Central Bureau of Statistics (CBS), UNICEF Sudan. 2016, Multiple Indicator Cluster Survey 2014 of Sudan, Final Report. Khartoum, Sudan: UNICEF and Central Bureau of Statistics (CBS), February 2016.

[18] UNICEF Somalia and Ministry of Planning and International Cooperation, 2014. Northeast Zone Multiple Indicator Cluster Survey 2011, Final Report. Nairobi, Kenya: UNICEF, Somalia and Ministry of Planning and International Cooperation.

[19] UNICEF Somalia and Somaliland Ministry of Planning and National Development, 2014. Somaliland Multiple Indicator Cluster Survey 2011, Final Report. Nairobi, Kenya: UNICEF, Somalia and Somaliland Ministry of Planning and National Development, Somaliland.

[20] Ministry of Public Health and Population (MOPHP), Central Statistical Organization (CSO) [Yemen], Pan Arab Program for Family Health (PAPFAM), and ICF International. 2015. Yemen National Health and Demographic Survey 2013. Rockville, Maryland, USA: MOPHP, CSO, PAPFAM, and ICF International.

[21] Bryce J, Requejo J. The Countdown 2008 report: Tracking progress in maternal, newborn, and child survival. NewYork: UNICEF; 2008.

[22] Stanton CK, Dubourg D, De Brouwere V, et al. Reliability of data on cesarean sections in developing countries. Bull World Health Organization 2005; 83(6):449- 455.

[23] Sevelsted A, Stokholm J, Bennelykke K, Bisgaard H. Cesarean section and chronic immune disorders. Pediatrics 2015; 135:e92-e98.

[24] Nhan T, Ho, Li F, Lee-Sarwar KA, Tun HM, Brown B, Paanaraj PS., et al. Meta-analysis of effects of exclusive breastfeeding on infant gut micorbiota across populations. Nature communications 9:4169.

[25] Stevens J, Schmied V, Burns E, Dahlen H. Immediate or early skin-to-skin contact after a Caesarean section: a review of the literature.Matern Child Nutr. 2014 Oct;10(4):456-73.

[26] World Health Report (2010). Background paper, 30. World Health organization (WHO), Geneva, Switzerland.

[27] Freitas PF, Drachler ML, Leite JC, Marshall T. Inequalities in cesarean delivery rates by ethnicity and hospital accessibility in Brazil. Int J Gynaecol Obstet. 2009;107(3):198201.https://doi.org/10.1016/j.ijgo.2009.08.017.

[28] Sakae TM, Freitas PF, D’Orsi E. Factors associated with cesarean section rates in a university hospital. Rev Saude Publica. 2009;43(3):472-80.

[29] Manyeh AK, Amu A., Akpakli DE, Williams J, Gyapong M. Socioeconomic and demographic factors associated with caesarean section delivery in Southern Ghana: evidence from INDEPTH Network member site. BMC Pregnancy and Childbirth (2018) 18:405.

[30] Preetkamal, HK, Nagpal M.Is current rising trends of cesarean section -justified? IJRCOG 2017 6(3): Online ISSN: 2320-1789.

[31] Dumont A, de Bernis L, Bouvier-Colle MH, Breart G, MOMA study group. Caesarean section rate for maternal indication in sub-Saharan Africa: a systematic review. Lancet 2001; 358 (9290): 132833.

[32] Mi J, Liu F. Rate of caesarean section is alarming in China. Lancet. 2014;383(9927):1463-4.

[33] Mittal S, Pardeshi S, Mayadeo N, Mane J. Trends in Cesarean Delivery: Rate and Indications. J Obstet Gynaecol India 2014;64(4):251-4.

[34] Al-Jawaldeh A, Abul-Fadl AM. Assessment of the Baby Friendly Hospital Initiative Implementation in the Eastern Mediterranean Region. Children 2018; 5(3):41. 
[35] Yazdizadeh B, Nedjat S, Mohammed K, Rashidan A, Changizi N, Majdzadeh R. Cesarean section rate in Iran, multi-dimensional approached for behavioural change of providers: A qualitative study . BMC Health Serv Res 2011;11:159.

[36] Lotfi R, Tehrani FM, Dovom MR, Torkestani F, Abedini M, Sajedinejad S. Development of strategies to reduce Cesarean delivery rates in Iran 2012-2014: A mixed methods study. In J Prev Med. 2014;5(12):1552-1566.

[37] Al Rifai R. Rising cesarean deliveries among apparently low-risk mothers at university teaching hospitals in Jordan: analysis of population survey data, 2002-2012. Glob Health Sci Pract. 2014;2(2):195-209.

[38] Al Rifai RH. Trend of cesarean deliveries in Egypt and its associated factors: evidence from national surveys, 2005-2014. BMC Preg \& Child birth. 2017;17:417.

[39] Althabe F, Sosa C, Belizán JM, Gibbons L, Jacquerioz F, Bergel E. Cesarean section rates and maternal and neonatal mortality in low-, medium-, and high income countries: an ecological study. Birth 2006; 33 (4): 270-7.

[40] Belizán JM, Althabe F, Cafferata ML. Health Consequences of the Increasing Caesarean Section Rates. Epidemiology 2007; 18 (4): 485-6.

[41] Faisal-Cury A, Menezes PR, Quayle J, Santiago K, Matijasevich A. The relationship between indicators of socioeconomic status and cesarean section in public hospitals. Rev Saude Publica 2017;51:14.

[42] Guilmoto CZ, Dumont A. Trends, Regional Variations, and Socioeconomic Disparities in Cesarean Births in India, 2010-2016. JAMA Netw Open. 2019;2(3):e190526. Published 2019 Mar 1. doi:10.1001/jamanetworkopen.2019.0526.

[43] World Health Organization. Appropriate technology for birth. Lancet. 1985;326(8452):436-7.

[44] Rowe-Murray HJ, Fisher JRW. Baby friendly hospital practices: cesarean section is a persistent barrier to early initiation of breastfeeding. Birth (Berkeley, Calif) 2002;29(2):124-31.

[45] WHO recommendations non-clinical interventions to reduce unnecessary caesarean sections ISBN 978-92-4-155033-8, Geneva: World Health Organization; 2018. License: CC BY-NC-SA 3.0 IGO.

[46] Meedya S, Fahy K, Kable A. Factors that positively influence breastfeeding duration to 6 months: a literature review. Women Birth 2010;23(4):135-45. 